EPJ Web of Conferences 82, 01046 (2015)

DOI: $10.1051 /$ epjconf/20158201046

(C) Owned by the authors, published by EDP Sciences, 2015

\title{
Study of natural convection in a freezer at different modes of operation of the refrigeration unit
}

\author{
Aleksander S. Krasnoshlykov and Vyacheslav I. Maksimov \\ Institute of Power Engineering, National research Tomsk Polytechnic University, Tomsk 634050, \\ Russia
}

\begin{abstract}
The paper discusses a study of natural convective in the volume of air and water in the freezer, under various operating conditions of the refrigeration unit. It was obtained changing the temperature of the water and air depending on the time of installation. It was revealed, that convective flows in a study area produce influence on the temperature field being frozen liquid. The values obtained by mathematical modelling are in good agreement with experimental data.
\end{abstract}

\section{Introduction}

Nowadays, a main part of industrial heat exchangers are ineffective. In their design were used the balance equation and the convective heat transfer processes were not taken [1]. With the increase need for energy conservation and resource efficiency of industrial enterprises is rising interest in the calculation of the influence of convective flows in free convection heat transfer unit [2-4].

Despite the huge number of papers devoted to the theory of convective heat transfer and its application, many problems of the theory studied is far enough, so the development of the theory of convective flows is of considerable theoretical and practical interest [5-8].

The aim of this work is the experimental determination of temperatures in the freezer refrigeration plant under natural convection and the presence of different thermal load.

\section{Experimental studies}

The experimental equipment is a heat exchanger freezer (Fig. 1), and consists of a four main parts: compressor, after the compressor is a condenser intended for the heat output of the heat exchanger the working fluid into the environment (no forced cooling). Next is the filter drier is designed to prevent pollution of the evaporator. One of the basic elements is a capillary tube heat exchanger designed for throttling of the working fluid and install differential pressure within the system. The evaporator is located directly in the freezer. Also the system is provided with a pressure gauge for detecting the pressure in the condenser and evaporator. In the freezer compartment and on the engine compressor

This is an Open Access article distributed under the terms of the Creative Commons Attribution License 4.0, which permits unrestricted use, distribution, and reproduction in any medium, provided the original work is properly cited. 


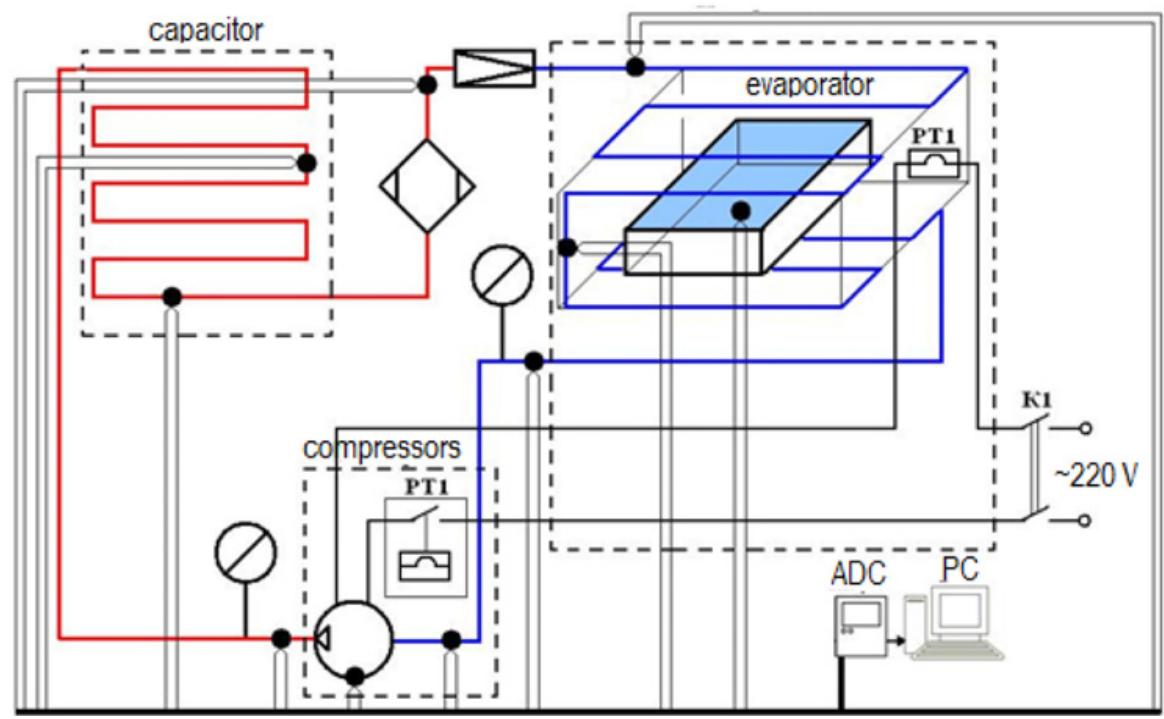

Figure 1. Schematic of the experimental equipment.

mounted thermal relay, so refrigeration unit can be taken out of service in two cases:

1). The temperature freezer reaches a certain value $\left(-15^{\circ} \div-25^{\circ} \mathrm{C}\right)$;

$2)$. Overheat the engine compressor $\left(\sim 90^{\circ}, \mathrm{C}\right)$.

The first case is more frequent and is considered normal operation so as the shutdown of the heat exchanger in first case is energy-saving action. Over temperature shutdown of the engine compressor are the results of abnormal operation of the refrigeration unit, which can be caused by several reasons. The main reason can be considered an unacceptable heat load in the freezer, which leads to the compressor operating without blackouts. Except overheating of the compressor may be caused by a lack of refrigerant in the system during its depressurization or violating the integrity of the electrical insulation of the motor windings.

Working body of the heat exchanger is the refrigerant R-134A.

The experiment was carried with freezer refrigeration unit (Fig. 2) $(0.5 \times 0.35 \times 0.25) \mathrm{m}$. In which there is a container of water $(0.3 \times 0.24 \times 0.1) \mathrm{m}$.

\section{Analysis of experimental results}

Figure 3 shows a graph of the temperature of the water and air from the time when the container was placed in a freezer at startup.

Analyzing the obtained temperature values for various operating modes can be concluded that the temperature fields freezable liquid product depends on the convective flow in the freezer. It was revealed that different conditions of work heat exchanger are not significantly affect the time of phase transition, because freezing the water occurred more quickly for economical mode when the container was placed in a freezer at startup. In the case of placing the volume water in a freezer when reached a stationary mode, the phase transition process is carried out quickly in area the lower boundary. This is because the container was placed on a cooled substrate evaporator, resulting occurred the intensification of heat transfer between the liquid and air in the freezer. 


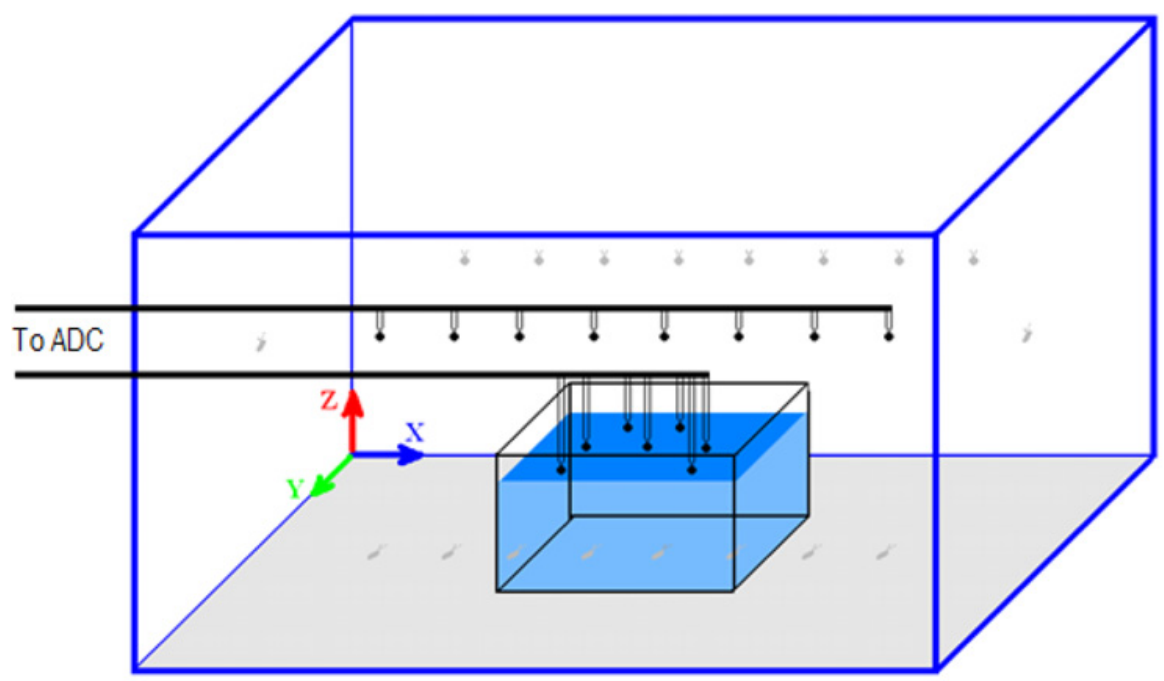

Figure 2. Schematic of the study area.

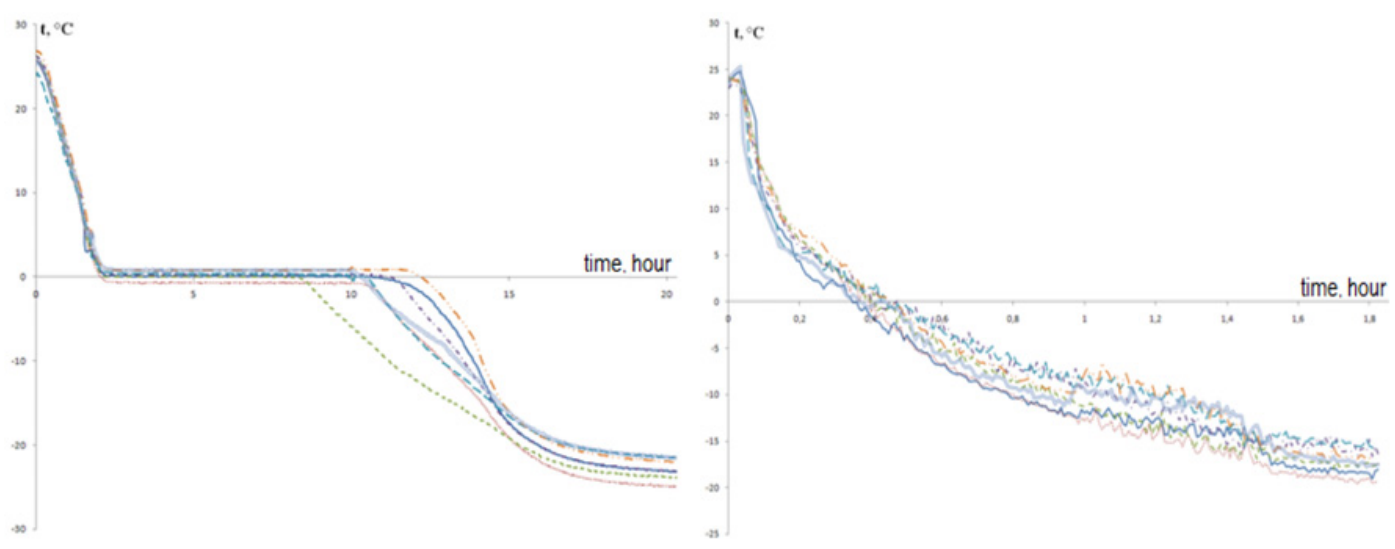

Figure 3. Changing the temperature of the water (left) and air (right) depending on the time of installation. -1 thermocouple; ........ 2 thermocouple; . -3 thermocouple; _ - $~-~-4$ thermocouple; $-5$ thermocouple; $=$. " - -6 thermocouple; -7 thermocouple.

The developed method of experiment and experimental equipment can used to further investigate this issue. At the same time, these data can be used to determine the optimum operating conditions of refrigerating units at different thermal loads.

\section{Mathematical modeling}

The mathematical model is a closed rectangular area with walls of finite thickness (Fig. 4). At the boundaries of the model are given boundary conditions of the second kind.

It was carried out a comparative analysis of the results obtained in the experimental study of convective flows in the freezer and temperature values obtained by solution of the problem of convective heat transfer in a rectangular area by mathematical modeling. As can be seen from the graph (Fig. 6), 


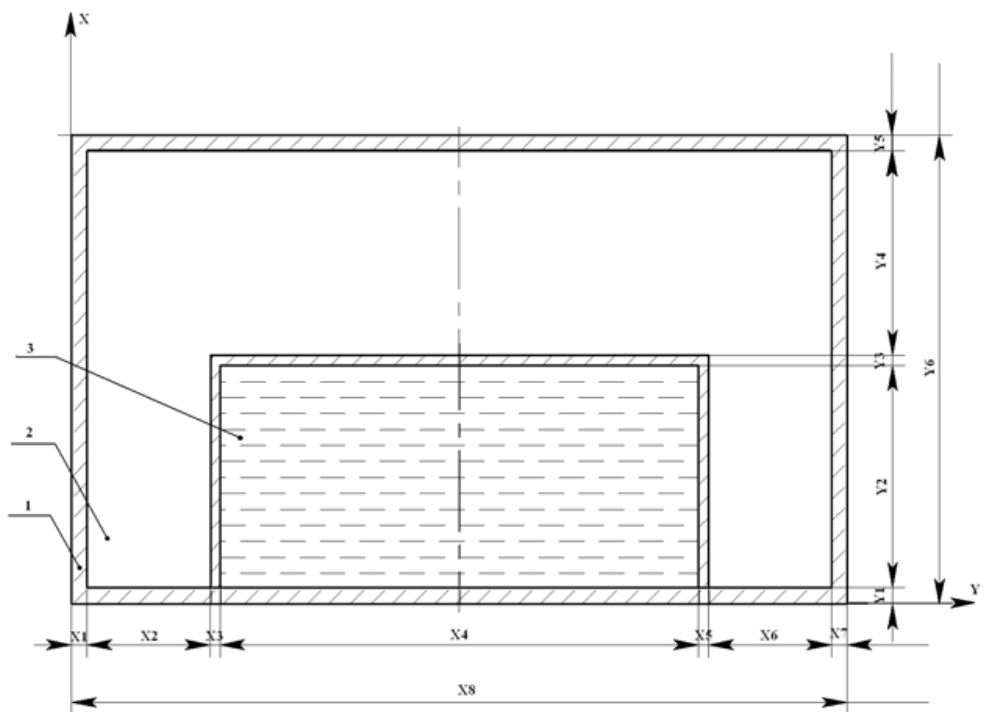

Figure 4. Mathematical model of the freezer with the heat load. 1-boundary, 2-air, 3-water.
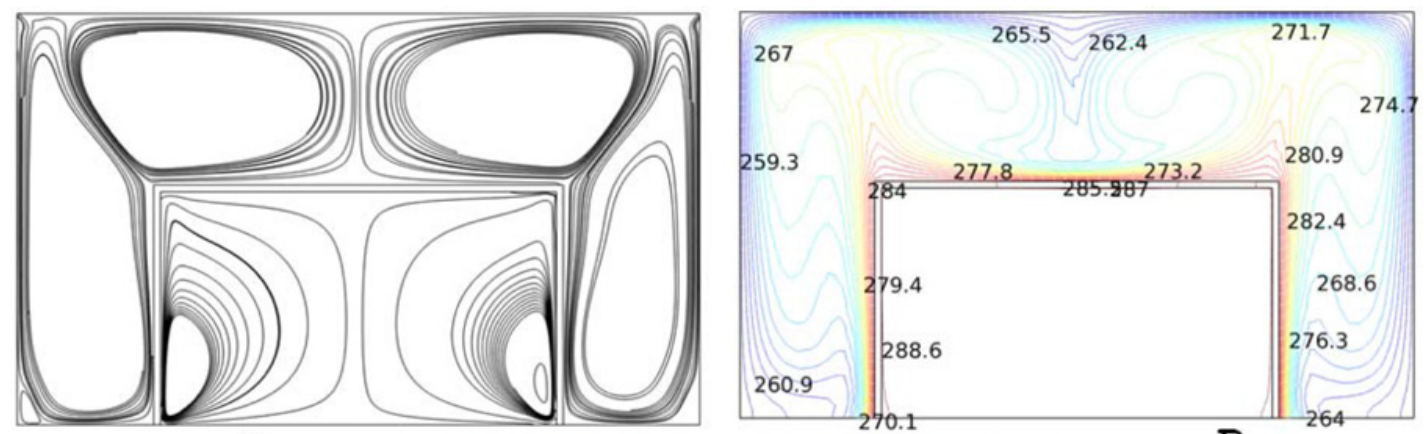

A

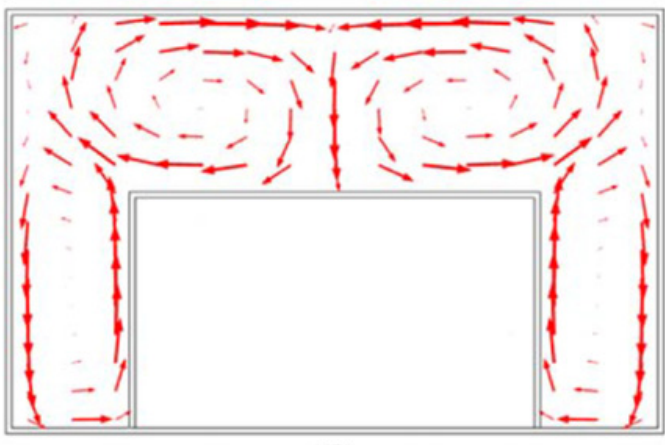

B

C

Figure 5. Isolines flow (A), the field distribution of temperature inside the study area (B), the structure of air masses in the test model $(\mathrm{C})$. 


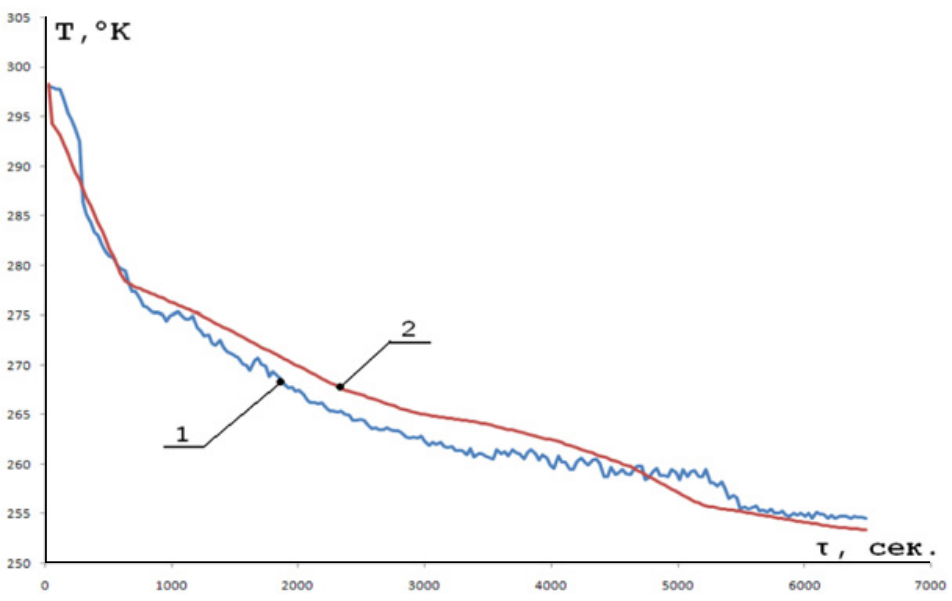

Figure 6. A graph of temperature values obtained experimentally (curve 1) and by means of mathematical modeling (curve 2).

the values obtained by mathematical modeling are in good agreement with experimental data. However, experimental research allows more thoroughly get the temperature field inside the freezer.

It was revealed that during operation of the refrigeration unit in the presence of heat load in the freezer occurs heterogeneity values temperature in various areas of the freezer. As a result of mathematical modeling of heat transfer processes in a closed volume revealed that the heterogeneity associated with the presence of intense convective flows of air and coolant in the freezer.

Relative error of the experimental study is $\delta \max =4 \%$. The maximum deviation of the results of mathematical modeling unlike experimental research is $\delta \max =5 \%$.

Work performed under the research state assignment "Science" (Code of Federal Target Scientific and Technical Program 2.1321.2014).

\section{References}

[1] G.V. Kuznetsov, V.I. Maksimov, M.A. Sheremet, Journal of Applied Mechanics and Technical Physics, 588-595 (2013)

[2] V.I. Maksimov, D.A. Nagornov, EPJ Web of Conferences, 4 (2014)

[3] G.V. Kuznetsov, V.I. Maksimov, Journal of Engineering Thermophysics, 270-275 (2007)

[4] Vladimír Gerlich, Kateřina Sulovská, Martin Zálešák, Measurement, 2003-2012 (2013)

[5] S.A. Ivanenko, P.P. Koryavov, Mathematical modeling 14, 6 (2002)

[6] G.V. Kuznetsov, V.I. Maksimov, Journal of Engineering Thermophysics 16, 4 (2007)

[7] G.V. Kuznetsov, N.I. Kurilenko, V.I. Maksimov, G.Ya. Mamontov, T.A. Nagornova, Journal of Engineering Physics and Thermophysics, 86, 3 (2013)

[8] G.V. Kuznetsov, M.A. Sheremet, Thermophysics and Aeromechanics 16, 1 (2009) 